\title{
Bacterial Causes of Infections and Their Antimicrobial Sensitivity Pattern in Cancer Patients
}

\author{
Dr. Md. Rafiqul Islam ${ }^{1 *}$, Dr. Farhana Akhter ${ }^{2}$, Professor Dr. Nazrina Khatun ${ }^{3}$, Dr. Mosfika Rahman ${ }^{4}$
}

\author{
${ }^{1}$ Assistant Professor, Department of Medical Oncology, National Institute of Cancer Research and Hospital (NICRH), Dhaka, \\ Bangladesh \\ ${ }^{2}$ Registrar, Department of Medical Oncology, National Institute of Cancer Research and Hospital (NICRH), Dhaka, Bangladesh \\ ${ }^{3}$ Head, Department of Medical Oncology, National Institute of Cancer Research and Hospital (NICRH), Dhaka, Bangladesh \\ ${ }^{4}$ Assistant Registrar, Department of Medical Oncology, National Institute of Cancer Research and Hospital (NICRH), Dhaka, \\ Bangladesh
}

\author{
Article History \\ Received: 21.01.2021 \\ Accepted: 03.03.2021 \\ Published: 12.03.2021 \\ Journal homepage: \\ https://www.easpublisher.com
}

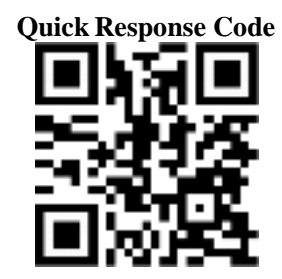

Abstract: Background: The increased risk of bacterial infections in the cancer patient is further compounded by the rising trends of antibiotic resistance in commonly implicated organisms. In Bangladesh the frequency of infections caused by E. coli, Klebsiella spp. and Proteus Spp. are high. Now a day the increasing resistance among several organisms is also a matter of concern. We have very few data regarding this issue. Aim of the study: The aim of this study was to document the bacterial causes of infections and describe their antimicrobial sensitivity pattern in cancer patients. Methods and Materials: This was a descriptive observational study, conducted in Department of Medical Oncology, National Institute of Cancer Research and Hospital, Dhaka, Bangladesh, during the period from January 2018 to December 2019. In total 19 cancer patients with bacterial infection were selected as study population. All cases were diagnosed from blood, urine, skin/soft tissue and respiratory samples i.e. cough swabs of patients. Samples were processed as per standard microbiology laboratory operating procedures. Socio-demographic and clinical data of respondents were collected using a structured questionnaire. Culture and antibiotic resistance were done following standard microbiological procedures. Results: According to culture reports we observed E. coli, Proteus, Klebsiella spp. and Pseudomonas spp. were associated in isolates formations in 53\%, 21\%, 16\% and $10 \%$ cases respectively. In analyzing the antimicrobial susceptibility among all participants we observed, against all causative organisms (E. coli, Proteus, Klebsiella and Pseudomonas) 94.74\% susceptibility had been shown by Ertapenem, Imipenem, Tobramycin, Fleroxacin Amikacin, Ceftriaxone, Cefuroxime, Nalidaxic acid, Dihydrofuran and Vancomycin. Then $89.47 \%$ susceptibility had been shown by Ceftriaxone, Doxycycline, Tetracycline, Levofloxacin, Ceftriaxone, Peniciline, Cotrimoxazole and Carbenicillin. Besides these, $94.74 \%$ susceptibility had been shown by $84.21 \%$ susceptibility had been shown by Amikacin, Gentamicin, Ampicillin, Cotrimoxazole and Piperacillin. On the other hand, in this study, more than $20 \%$ resistance was found against Ciprofloxacin, Vancomycin, Cefepime, Cefixime, Ceftazidime, Azithromycin, Aztreonam, Cefotaxime, Amoxicillin, Ceftriaxone, Meropenem and Amoxyclav. Conclusion: Ertapenem, Imipenem, Tobramycin, Fleroxacin, Amikacin, Ceftriaxone, Cefuroxime, Nalidaxic acid, Dihydrofuran and Vancomycin, Ceftriaxone, Doxycycline, Tetracycline, Levofloxacin, Ceftriaxone, Peniciline, Cotrimoxazole and Carbenicillin. Amikacin, Gentamicin, Ampicillin, Cotrimoxazole and Piperacillin may be considered as the best antibiotics against E. coli, Proteus, Klebsiella and Pseudomonas infections in cancer patients.

Keywords: Bacterial causes, cancer patients, infections, antimicrobial sensitivity.

Copyright () 2021 The Author(s): This is an open-access article distributed under the terms of the Creative Commons Attribution 4.0 International License (CC BY-NC 4.0) which permits unrestricted use, distribution, and reproduction in any medium for non-commercial use provided the original author and source are credited.

\section{INTRODUCTION}

Basically, the increased risk of bacterial infections in the cancer patient is further compounded by the rising trends of antibiotic resistance in commonly implicated organisms. In Bangladesh this is particularly true in case of Gram negative bacilli such as E. coli,
Klebsiella spp. and Acinetobacter spp. Now a day the increasing resistance among Gram positive organisms is also a matter of concern. Till now in spite of vast advances made by medical science in cancer treatment, infections remain a major cause of morbidity and mortality in patients diagnosed with cancer. The cancer patient is immunocompromised because of the nature of 
the disease itself and also due to interventions in the form of chemotherapy, immune therapy etc., in addition, there are usually other associated risk factors for acquiring infection such as long term catheterization, mucositis due to cytotoxic agents, neutropenia, and stem cell transplantation [1]. This increased risk of bacterial infections is further compounded by the rising trends of antibiotic resistance in commonly implicated organisms all over the world. This is particularly true in the case of members of Enterobacteriaceae group like Escherichia coli and Klebsiella pneumoniae and the nonfermenter group of organisms such as Acinetobacter spp. In the Indian setting. There is already widespread resistance to the cephalosporins as shown by ESBL (extended spectrum $\beta$-lactamase) and Ampicillin producers among the Enterobacteriaceae [2]. Rampant use of antibiotics has unfortunately led to increasing resistance to the carbapenems as well, and this is generally due to carbapenemase production by the organisms [3]. Prevalence of Metallo- $\beta$-lactamase (MBL) producing organisms including New Delhi MBL-1 (NDM-1) is also on the rise in India [4]. Increasing resistance among Gram-positive organisms is also a matter of concern. High rates of Methicillin-Resistant Staphylococcus aureus (MRSA) in clinical samples have been noted in one study from North East India [5]. Similarly, resistance to the glycopeptide antibiotics such as vancomycin and tiecoplanin among clinical isolates of enterococci is also increasing [6]. The symptoms of infection in cancer patients could be masked by the cancer treatment modalities [7] that are an indicator for considering asymptomatic infections. Previous studies on bacterial infection and drug resistance pattern among cancer patients were mainly focused on bloodstream infection (BSI) with hematologic malignancies [7]. Cancer patients who have solid tumors might have a tendency to undergo surgery to remove the tumor or sometimes due to other medical reasons. This increases the potential of acquiring bacterial infection either by endogenously normal flora near the operative sites or exogenously from the hospital environments, such as in the air, hospital staff, inanimate objects, and medical equipment, as a result of their prolonged and frequent contact [8]. Therefore, patients with both type of cancer are highly susceptible to almost any type of bacterial infection [9]. Among Gram positive bacteria (GPB) genus Staphylococcus and from Gram negative bacteria (GNB): Escherichia coli, Klebsiella pneumoniae, and Pseudomonas aeruginosa are frequently associated [10]. Moreover, frequent prescription of broad-spectrum antibiotics as prophylaxis among cancer patients may potentially alter the composition of endogenous flora and select multidrug resistant pathogens such as methicillin-resistant Staphylococcus aureus (MRSA), vancomycin-resistant enterococci (VRE), and Gramnegative bacilli (eg, fluoroquinolone-resistant Escherichia coli [FREC]) [11]. As a result, empirical antibiotic treatments of cancer patients are continually challenged by the change in frequency of Gram-positive as well as Gram-negative bacteria and the emergence of new antimicrobial resistant pathogens. The pattern and prevalence of resistance may vary with respect to geographical location and difference in infection prevention as well as control strategies between health care facilities. The treatment of bacterial infections in patients with cancer should often rely on the use of established guidelines, along with consideration of the local epidemiology and antibiotic susceptibility patterns of the potential etiologic agents.

\section{OBJECTIVES \\ General Objective}

- To document the bacterial causes of infections and describe their antimicrobial sensitivity pattern in cancer patients.

\section{Specific Objective}

- To collect information regarding the sociodemographic characteristics of cancer patients.

- To collect information regarding the clinical characteristics of cancer patients.

- To collect information regarding the antimicrobial sensitivity on several causative organisms in cancer patients.

\section{Methodology \& Materials}

This was a descriptive observational study and it was conducted in Department of Medical Oncology, National Institute of Cancer Research and Hospital, Dhaka, Bangladesh, during the period from January 2018 to December 2019. In total 19 cancer patients with several bacterial infections attended the mentioned hospital with proper documentation were selected as study population. The age range of the participants was 18-60 years. All cases as well as isolates were diagnosed from blood, urine, skin/soft tissue and respiratory samples i.e. cough swabs of patients. Samples were processed as per standard microbiology laboratory operating procedures. Socio-demographic and clinical data of respondents were collected using a structured questionnaire. Culture and antibiotic resistance were done following standard microbiological procedures. The study was reviewed and approved by the ethical committee of the mentioned hospital. Proper writtenconsents were taken from all the participants before starting data collection. Clinical examination and other co-morbidity factors were diagnosed by an oncologist. Socio-demographic and clinical data were collected by using a structured questionnaire by nurses working in the center. According to the patients' clinical status, different types of specimen were collected. The blood samples were collected, processed and transferred into culture bottles of sterile tryptic soy broth (Oxoid Ltd., Basingstoke, UK). Bottles were incubated at $37^{\circ} \mathrm{C}$ for 7 days and observed for signs of bacterial growth (turbidity, hemolysis, clot formation) on a daily basis for up to7 days Bottles which showed

(C) East African Scholars Publisher, Kenya 
signs of growth were gram stained and sub cultured on blood agar, chocolate agar, Mac Conkey agar, and mannitol salt agar. These plates were than aerobically incubated for $18-24$ hours at $37^{\circ} \mathrm{C}$. Blood sample containing broths with no bacterial growth after 7 days was sub-cultured before being reported as a negative result. Absolute neutrophil count was done using a XT4000i hematology analyzer (Sysmex Europe $\mathrm{GmbH}$, Norderstedt, Germany). Midstream urine of the participants was collected with a sterile urine container from both symptomatic and asymptomatic urinary tract infection (UTI) cases. Midstream urine specimens were inoculated on cystine lactose electrolyte deficient (CLED) by using a calibrated loop $(0.001 / \mathrm{mL})$. All the media were incubated at $37^{\circ} \mathrm{C}$ for $18-24$ hours. Significant bacteriuria was defined as colony count $\geq 10^{5} \mathrm{CFU} / \mathrm{mL}$ urine. The swabs/ear discharges of the patients were streaked on Mac-Conkey agar, chocolate agar, blood agar plates, and mannitol salt agar. These plates were then aerobically incubated for 18-24 hours at $37^{\circ} \mathrm{C}$. The bacterial pathogens among patients were identified after appearance of growth on subcultured/cultured plates of blood/wound swab/discharge samples and significant growth on CLED by standard microbiological and biochemical procedures [12]. Antibiotic susceptibility testing of bacterial isolates was done by Kirby Bauer disc diffusion method using Muller Hinton agar (MHA) plate (Oxoid Ltd.) [13]. All the necessary data were collected, coded, analyzed and disseminated by using MS Office and SPSS software version 20 .

\section{RESULTS}

In this study, a total of 19 cancer patients were included in the study. Out of these, $11(57.59 \%)$ were male and $8(42.11 \%)$ were female, with a male-female ratio of a $1.38: 1$. The mean age $( \pm S D)$ of study participants was $42.21 \pm 15.9$ years, range $18-71$ years. In this study, the highest number of patients was found from 41-60 years' age group which was $47.37 \%$. Then $36.84 \%$ and $15.79 \%$ patients were found from 10-40 and $>60$ years' age groups respectively. On the other hand, in diagnosis procedure Ca Lt. Breast (Recurrence Local) and adenoma were found in $15.79 \%$ patients separately. Then in $10.53 \%$ patients $\mathrm{Ca}$ Rectum, Ewing's Sarcoma, Ca Lung (Rt) and Liver metastasis were found separately. Moreover, in $5.26 \%$ patients $\mathrm{Ca}$ Buccal mucosa, Ca Cervix, Ca Left lung, Ca Rt middle ear, Fibrosarcoma, Osteosarcoma, Ca Rt. Breast and Ca Lung (Lt) were involved separately. Besides these, fever, including surgical, wounds and ports, change in cough or new cough and unusual vaginal discharge or irritation were found as present complaints in $10.53 \%$, another $10.53 \%, 5.26 \%$ and in another $5.26 \%$ respectively. Besides these complaints, some other complaints were associated in $21 \%$ participants. In this study among all the participants only $26.32 \%$ participant gave history of taking antibiotics was found. In this study among all the participants the mean $( \pm \mathrm{SD})$ $\mathrm{Hb} \%, \mathrm{RBC}, \mathrm{WBC}$ and Platelet counts were $10.84 \pm 1.67$, $3.49 \pm 0.00,7713.79 \pm 5868.75$ and $144807.25 \pm 129325.25$ respectively. Besides these, in analyzing the differential count we found, the mean $( \pm \mathrm{SD})$ Neutrophil, Lymphocyte, Basophil and Monocyte were $58.17 \pm 23.50,22.71 \pm 18.36, \quad 1.71 \pm 3.05$ and $2.64 \pm 1.79$ respectively. In this study according to culture reports we observed E. coli, Proteus, Klebsiella spp. and Pseudomonas spp. were associated in isolates formations in $53 \%, 21 \%, 16 \%$ and $10 \%$ cases respectively. In analyzing the antimicrobial susceptibility among all participants we observed, against all causative organisms (E. coli, Proteus, Klebsiella and Pseudomonas) 94.74\% susceptibility had been shown by Ertapenem, Imipenem, Tobramycin, Fleroxacin Amikacin, Ceftriaxone, Cefuroxime, Nalidaxic acid, Dihydrofuran and Vancomycin. Then $89.47 \%$ susceptibility had been shown by Ceftriaxone, Doxycycline, Tetracycline, Levofloxacin, Ceftriaxone, Peniciline, Cotrimoxazole and Carbenicillin. Besides these, $84.21 \%$ susceptibility had been shown by Amikacin, Gentamicin, Ampicillin, Cotrimoxazole and Piperacillin. On the other hand, in this study, more than $20 \%$ resistance was found against Ciprofloxacin, Vancomycin, Cefepime, Cefixime, Ceftazidime, Azithromycin, Aztreonam, Cefotaxime, Amoxicillin, Ceftriaxone, Meropenem and Amoxyclav.

Table-I: Demographic characteristics of participants $(\mathrm{N}=19)$

\begin{tabular}{|l|c|c|}
\hline Characteristics & n & \% \\
\hline Age (years) & 9 & 47.37 \\
\hline 18-40 Years & 7 & 36.84 \\
\hline 41-60 Years & 3 & 15.79 \\
\hline >60 Years \\
\hline Sex & 11 & 57.89 \\
\hline Male & 8 & 42.11 \\
\hline Female
\end{tabular}


Table-II: Clinical and histopathological characteristics of participants $(\mathrm{N}=19)$

\begin{tabular}{|c|c|c|}
\hline \multirow[b]{2}{*}{ Characteristics } & \multirow[b]{2}{*}{$\mathbf{n}$} & \multirow[b]{2}{*}{$\%$} \\
\hline & & \\
\hline \multicolumn{3}{|l|}{\begin{tabular}{|c|} 
Diagnosis \\
\end{tabular}} \\
\hline Ca Lt. Breast (Recurrence Local) & 3 & 15.79 \\
\hline Adenoma of parotid gland & 3 & 15.79 \\
\hline Ca Rectum & 2 & 10.53 \\
\hline Ewing's Sarcoma & 2 & 10.53 \\
\hline Ca Right Lung & 2 & 10.53 \\
\hline Liver metastasis & 2 & 10.53 \\
\hline Ca Buccal mucosa & 1 & 5.26 \\
\hline Ca Cervix & 1 & 5.26 \\
\hline Ca Left Lung & 1 & 5.26 \\
\hline Ca Rt middle ear & 1 & 5.26 \\
\hline Fibrosarcoma & 1 & 5.26 \\
\hline Osteosarcoma & 1 & 5.26 \\
\hline Ca Rt. Breast & 1 & 5.26 \\
\hline \multicolumn{3}{|c|}{ Present complaints } \\
\hline Fever & 2 & 10.53 \\
\hline Including surgical wounds and ports & 2 & 10.53 \\
\hline Change in cough or new cough & 1 & 5.26 \\
\hline Unusual vaginal discharge or irritation & 1 & 5.26 \\
\hline Others & 4 & 21.05 \\
\hline \multicolumn{3}{|c|}{ History of taking antibiotics } \\
\hline Present & 5 & 26.32 \\
\hline Absent & 14 & 73.68 \\
\hline \multicolumn{3}{|l|}{ Sample } \\
\hline Pus & 7 & 36.84 \\
\hline Urine & 5 & 26.32 \\
\hline Discharge from any sites & 4 & 21.05 \\
\hline Sputum & 2 & 10.53 \\
\hline Others & 1 & 5.26 \\
\hline \multicolumn{3}{|c|}{ Complete blood count $($ Mean \pm SD) } \\
\hline Mean $\mathrm{Hb \%}$ & & $10.84 \pm 1.67$ \\
\hline Mean RBC & & $3.49 \pm 0.00$ \\
\hline Mean WBC & & $13.79 \pm 5868.75$ \\
\hline Mean Platelet & 1448 & $.25 \pm 129325.25$ \\
\hline \multicolumn{3}{|c|}{ Differential Count $($ Mean \pm SD) } \\
\hline Mean Neutrophil & & $58.17 \pm 23.50$ \\
\hline Mean Lymphocyte & & $22.71 \pm 18.36$ \\
\hline Mean Basophil & & $1.71 \pm 3.05$ \\
\hline Mean Monocyte & & $2.64 \pm 1.79$ \\
\hline
\end{tabular}

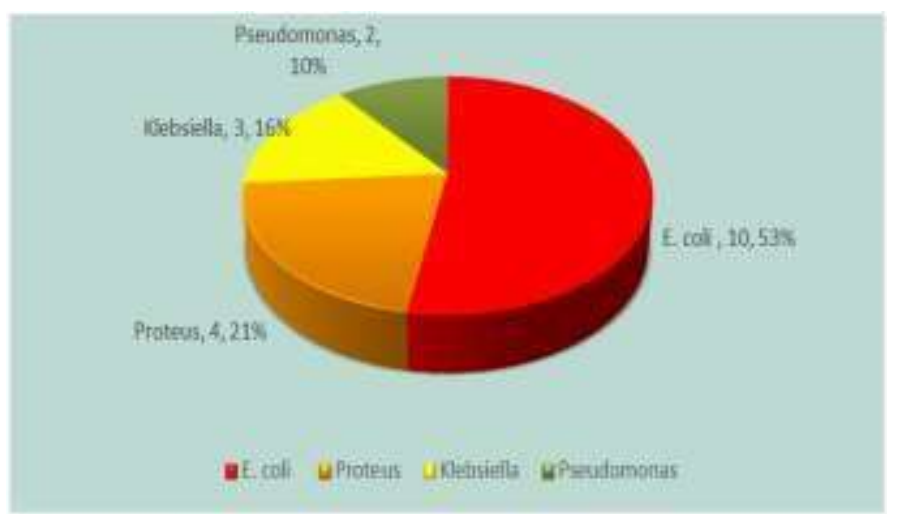

Fig-I: Antimicrobial susceptibility among all participants (N=19) 
Table-III: Antimicrobial susceptibility among all participants $(\mathrm{N}=19)$

\begin{tabular}{|l|c|c|}
\hline Antimicrobial agents & n & \% \\
\hline Ertapenem & 18 & 94.74 \\
\hline Imipenem & 18 & 94.74 \\
\hline Tobramycin & 18 & 94.74 \\
\hline Fleroxacin & 18 & 94.74 \\
\hline Amikacin & 18 & 94.74 \\
\hline Ceftriaxone & 18 & 94.74 \\
\hline Cefuroxime & 18 & 94.74 \\
\hline Nalidaxic acid & 18 & 94.74 \\
\hline Dihydrofuran & 18 & 94.74 \\
\hline Vancomycin & 18 & 94.74 \\
\hline Ceftriaxone & 17 & 89.47 \\
\hline Doxycycline & 17 & 89.47 \\
\hline Tetracycline & 17 & 89.47 \\
\hline Levofloxacin & 17 & 89.47 \\
\hline Peniciline & 17 & 89.47 \\
\hline Cotrimoxazole & 17 & 89.47 \\
\hline Carbenicillin & 17 & 89.47 \\
\hline Gentamicin & 16 & 84.21 \\
\hline Ampicillin & 16 & 84.21 \\
\hline Piperacillin & 16 & 84.21 \\
\hline Ciprofloxacin & 15 & 78.95 \\
\hline Cefepime & 15 & 78.95 \\
\hline Cefixime & 15 & 78.95 \\
\hline Ceftazidime & 14 & 73.68 \\
\hline Azithromycin & 14 & 73.68 \\
\hline Aztreonam & 14 & 73.68 \\
\hline Cefotaxime & 14 & 73.68 \\
\hline Amoxicillin & 14 & 73.68 \\
\hline Meropenem & 12 & 63.16 \\
\hline Amoxyclav & 11 & 57.89 \\
\hline & & \\
\hline
\end{tabular}

\section{DISCUSSION}

The aim of this study was to document the bacterial causes of infections and describe their antimicrobial sensitivity pattern in cancer patients. In our study according to culture reports we observed E. coli, Proteus, Klebsiella spp. and Pseudomonas spp. were associated in isolates formations in $53 \%, 21 \%$, $16 \%$ and $10 \%$ cases respectively. In this study association of Gram positive bacteria was not found. But in a study, chemotherapy-induced mucositis and use of both prophylactic and empiric antibiotic regimens targeting GNB diminishes recovery of Gramnegative pathogens, while selecting for GPB were reported [14]. In contrary, a study from other African countries reported that GNB were significantly more predominant isolates from cancer patients [10], and current data from other studies indicates the reemergence of GNB among febrile neutropenic cancer patients [11]. This might be due to minimal use or the discontinuation of fluoroquinolones prophylaxis [15].
Table-IV: Antimicrobial resistance among all participants $(\mathrm{N}=19)$

\begin{tabular}{|l|c|c|}
\hline Antimicrobial agents & $\mathbf{n}$ & $\mathbf{\%}$ \\
\hline Ertapenem & 1 & 5.26 \\
\hline Imipenem & 1 & 5.26 \\
\hline Tobramycin & 1 & 5.26 \\
\hline Fleroxacin & 1 & 5.26 \\
\hline Amikacin & 1 & 5.26 \\
\hline Ceftriaxone & 1 & 5.26 \\
\hline Cefuroxime & 1 & 5.26 \\
\hline Nalidaxic acid & 1 & 5.26 \\
\hline Dihydrofuran & 1 & 5.26 \\
\hline Vancomycin & 1 & 5.26 \\
\hline Doxycycline & 2 & 10.53 \\
\hline Tetracycline & 2 & 10.53 \\
\hline Levofloxacin & 2 & 10.53 \\
\hline Peniciline & 2 & 10.53 \\
\hline Cotrimoxazole & 2 & 10.53 \\
\hline Gentamicin & 3 & 15.79 \\
\hline Ampicillin & 3 & 15.79 \\
\hline Piperacillin & 3 & 15.79 \\
\hline Ciprofloxacin & 4 & 21.05 \\
\hline Cefepime & 4 & 21.05 \\
\hline Cefixime & 4 & 21.05 \\
\hline Ceftazidime & 5 & 26.32 \\
\hline Azithromycin & 5 & 26.32 \\
\hline Aztreonam & 5 & 26.32 \\
\hline Cefotaxime & 5 & 26.32 \\
\hline Amoxicillin & 5 & 26.32 \\
\hline Meropenem & 7 & 36.84 \\
\hline Amoxyclav & 8 & 42.11 \\
\hline & \\
\hline
\end{tabular}

These findings strengthen the need for frequent surveillance for understanding the local epidemiology of bacterial infection among cancer patients. S. aureus, Coagulase-negative staphylococci(CoNS), and E. coli were the most common bacterial pathogens isolated in cancer patients in this study. More or less similar patterns have been reported in cancer patients in different countries, although the proportion of the bacterial agents varied [16]. The GPB S. aureus and Coagulase-negative staphylococci(CoNS) are ubiquitous in nature, which are frequently found on the skin and are the main cause of various infections, mainly in patients with solid tumors following indwelling devices, invasive surgical procedures, and contamination from hospital environments [17]. Likewise, $E$. coli is a normal member of gastrointestinal flora and a common cause of both community and hospital acquired UTI. Particularly immunocompromised cancer patients are easily colonized by the bacteria, due to the fact that infection of cancer patients by this bacterium is inevitable. In our study, in analyzing the antimicrobial susceptibility among all participants we observed, 
against all causative organisms (E. coli, Proteus, Klebsiella and Pseudomonas) $94.74 \%$ susceptibility had been shown by Ertapenem, Imipenem, Tobramycin, Flurloxacin, Amikacin, Ceftriaxone, Cefuroxime, Nalidaxic acid, Dihydrofuran and Vancomycin. Then $89.47 \%$ susceptibility had been shown by Ceftriaxane, Doxymycin, Tetracycline, Levofloxacin, Ceftriaxone, Peniciline, Cotrimoxazole and Carbenicillin. Besides these, $84.21 \%$ susceptibility had been shown by Amikacin, Gentamicin, Ampicillin, Cotrimoxazole and Piperacillin. On the other hand, in this study, more than $20 \%$ resistance was found against Ciprofloxacin, Vancomycin, Cefepime, Cefixime, Ceftazidime, Azithromycin, Aztreonam, Cefotaxime, Amoxicillin, Ceftriaxone, Meropenem and Amoxyclav. Although the development of MDR is a natural phenomenon, an interestingly extensive raise in the number of immunocompromised conditions, like cancer, increases MDR, thereby contributing to a further spread of MDR isolates, since these patients had frequent follow-up within the hospital [17]. In this study, the overall prevalence of MDR was $45 \%$ lower than a recent report in the same place from neonatal septicemia (65\%) [18]. Among the isolated bacteria, K. pneumoniae and E. coli were the principal MDR strains concordant with a previous study in the same place [18]. Most studies have shown that cancer patients with hematological malignancy had a higher risk of developing bacterial infection as compared to solid tumor patients [19].

\section{CONCLUSION AND \\ RECOMMENDATIONS}

In this study the rate of Gram +ve isolates was found higher than Gram -ve isolates. But most of the antimicrobial agents showed satisfactory susceptibility against GPB isolates. On the other hand, a noticeable number of antimicrobial agents had failed to show satisfactory susceptibility against GNB isolates. So we can conclude GNB isolates are difficult to treat by generally used antimicrobial agents. On the other hand, E. coli had been proved as the most notorious bacteria to treat. This was a single centered study with a small sized sample. So the findings of this study may not reflect the exact scenario of the whole community. So for getting more specific findings we would like to recommend for conducting more studies regarding the same issue.

\section{REFERENCES}

1. Bhat, V., Gupta, S., Kelkar, R., Biswas, S., Khattry, N., Moiyadi, A., \& Gupta, T. (2016). Bacteriological profile and antibiotic susceptibility patterns of clinical isolates in a tertiary care cancer center. Indian journal of medical and paediatric oncology: official journal of Indian Society of Medical \& Paediatric Oncology, 37(1), 20.
2. Rudresh, S. M., \& Nagarathnamma, T. (2011) Extended spectrum $\beta$-lactamase producing Enterobacteriaceae \& antibiotic co-resistance. The Indian journal of medical research, 133(1), 116.

3. Tzouvelekis, L. S., Markogiannakis, A., Psichogiou, M., Tassios, P. T., \& Daikos, G. L. (2012). Carbapenemases in Klebsiella pneumoniae and other Enterobacteriaceae: an evolving crisis of global dimensions. Clinical microbiology reviews, 25(4), 682-707.

4. Kumar, S. G., Adithan, C., Harish, B. N., Sujatha, S., Roy, G., \& Malini, A. (2013). Antimicrobial resistance in India: A review. Journal of natural science, biology, and medicine, 4(2), 286.

5. Tsering, D. C., Pal, R., \& Kar, S. (2011). Methicillin-resistant Staphylococcus aureus: Prevalence and current susceptibility pattern in Sikkim. Journal of Global infectious diseases, 3(1), 9.

6. Muto, C. A., Jernigan, J. A., Ostrowsky, B. E., Richet, H. M., Jarvis, W. R., Boyce, J. M., \& Farr, B. M. (2003). SHEA guideline for preventing nosocomial transmission of multidrug-resistant strains of Staphylococcus aureus and enterococcus. Infection control and hospital epidemiology, 24(5), 362-386.

7. Greene, J.N. (2004). Cheson BD. Infections in Cancer Patients (Basic and Clini- cal Oncology). 5th ed. New York: Marcel Dekker, Inc.

8. Homsi, J., Walsh, D., Panta, R., Lagman, R., Nelson, K. A., \& Longworth, D. L. (2000). Infectious complications of advanced cancer. Supportive care in cancer, 8(6), 487-492.

9. Thom KA, Kleinberg M, Roghmann M. Infection Prevention in the Cancer Center. Clin Infect Dis. 2013:57(4):579-585.

10. Zorgani, A. A., Belgasim, Z., Ziglam, H., \& Ghenghesh, K. S. (2012). Antimicrobial susceptibility profiles of gram-negative bacilli and gram-positive cocci isolated from cancer patients in Libya. Archives of Clinical Microbiology, 3(3).

11. Markman, M. (2011). Principles and Practice of Cancer Infectious Diseases. Lubbock, $T X$ : Humana press.

12. Cheesbrough, M. (2006). District Laboratory Practice in Tropical Countries: Part 2. 2nd ed. New York: Cambridge University, 1-43.

13. Bayer, A. W., Kirby, W. M. M., Sherris, J. C., \& Turck, M. (1966). Antibiotic susceptibility testing by a standardized single disc method. Am J clin pathol, 45(4), 493-496.

14. Bow, E. J. (2011). Fluoroquinolones, antimicrobial resistance and neutropenic cancer patients. Current opinion in infectious diseases, 24(6), 545-553.

15. Montassier, E., Batard, E., Gastinne, T., Potel, G., \& de La Cochetière, M. F. (2013). Recent changes in bacteremia in patients with cancer: a systematic review of epidemiology and antibiotic resistance. European journal of clinical 
microbiology \& infectious diseases, 32(7), 841850.

16. Almaziny, M. A. (2014). Isolation, identification, and profile of antibiotic resistance of bacteria in childhood febrile neutropenia patients. Eur J Exp Biol, 4(2), 1-6.

17. Tanwar, J., Das, S., Fatima, Z., \& Hameed, S. (2014). Multidrug resistance: an emerging crisis. Interdisciplinary perspectives on infectious diseases, 2014.
18. Moges, F., Eshetie, S., Yeshitela, B., \& Abate, E. (2017). Bacterial etiologic agents causing neonatal sepsis and associated risk factors in Gondar, Northwest Ethiopia. BMC pediatrics, 17(1), 1-10.

19. Sirkhazi, M., Sarriff, A., Abd Aziz, N., Almana, F., Arafat, O., \& Shorman, M. (2014). Bacterial spectrum, isolation sites and susceptibility patterns of pathogens in adult febrile neutropenic cancer patients at a specialist hospital in Saudi Arabia. World journal of oncology, 5(5-6), 196.

Cite This Article: Rafiqul Islam et al (2021). Bacterial Causes of Infections and Their Antimicrobial Sensitivity Pattern in Cancer Patients. EAS J Radiol Imaging Technol, 3(2), 29-35. 